\title{
Pharmacokinetic considerations on the repurposing of ivermectin for treatment of COVID-19
}

\author{
Ricardo Pena Silva ${ }^{1}$, Stephen Duffull ${ }^{2}$, Andrew Steer ${ }^{3}$, Sandra Jaramillo Rincon ${ }^{1}$, Amanda \\ Gwee $^{4}$, and Xiao Zhu ${ }^{2}$ \\ ${ }^{1}$ Universidad de los Andes \\ ${ }^{2}$ University of Otago \\ ${ }^{3}$ Murdoch Childrens Research Institute \\ ${ }^{4}$ The Royal Children's Hospital Melbourne
}

April 29, 2020

Hundreds of researchers are working to develop a vaccine and are evaluating drugs to mitigate the adverse health and economic consequences of COVID-19 (Coronavirus disease 19) worldwide. If novel compounds are found, geopolitical and economic variables will determine their introduction to communities. Therefore, finding low-cost and widely accessible drugs for prevention or treatment of COVID-19 would be ideal.

A recent study found that ivermectin, an FDA-approved anti-parasitic drug, has inhibitory effects on replication of the severe acute respiratory syndrome coronavirus $2(\mathrm{SARS}-\mathrm{CoV}-2)^{1}$. Ivermectin has broad anti-viral activity through inhibition of viral proteins including importin $\alpha / \beta 1$ heterodimer and integrase protein ${ }^{2}$. Caly and colleagues reported that the addition of ivermectin at a concentration of 5 micromolar $(\mu \mathrm{M})$ (twice the reported IC50) to Vero-hSLAM cells, 2 hours post infection with SARS-CoV-2, resulted in a reduction in the viral RNA load by $99.98 \%$ at 48 hours $^{1}$. The authors suggested that this drug could reduce the viral load in infected patients, with potential effect on disease progression and spread.

While the findings by Caly and colleagues provide some promise, there is no evidence that the $5 \mu \mathrm{M}$ concentration of ivermectin used by Caly and colleagues in their in vitro SARS-CoV-2 experiment, can be achieved in vivo. The pharmacokinetics of ivermectin in humans is well described (Figure 1) ${ }^{3-5}$, and even with the highest reported dose of approximately $1700 \mu \mathrm{g} / \mathrm{kg}$ (i.e. 8.5 times the FDA-approved dose of $200 \mu \mathrm{g} / \mathrm{kg}$ ), the maximum plasma concentration was only $0.28 \mu \mathrm{M}^{5}$. This is 18 times lower than the concentration required to reduce viral replication of SARS-CoV-2in vitro. Ivermectin accumulation in tissues is mild and would not be sufficient to achieve the antiviral effect with conventional doses ${ }^{6}$. Although high doses of ivermectin in adults or children are well tolerated ${ }^{5,7}$, the clinical effects of ivermectin at a concentration of $5 \mu \mathrm{M}$ range are unknown and may be associated with toxicity. Consequently, ivermectin has in vitroactivity against SARS-CoV-2 but this effect is unlikely to be observedin vivo using current dosing.

Amidst fear of the pandemic, the public and some physicians are now using ivermectin off-label for prophylaxis or as adjuvant therapy for COVID-19. Because ivermectin is only commercially available as a 3 or $6 \mathrm{mg}$ tablets or a $6 \mathrm{mg} / \mathrm{ml}$ oral suspension, in order to administer a high dose, some people may experiment with more concentrated veterinary formulations. These actions are not based on clinical trials and have motivated cautionary statements from institutions such as the FDA against the use of pharmaceutical formulations of ivermectin intended for animals as therapeutics in humans ${ }^{8}$.

Potential avenues for further investigation into repurposing ivermectin for SARS-CoV-2 may be to: (i) develop an inhaled formulation to efficiently deliver a high local concentration in the lung, whilst minimizing systemic exposure; and (ii) evaluate more potent ivermectin analogs (e.g. doramectin) which may also inhibit 
SARS-CoV-2. These are areas for research - clearly, further studies are needed before ivermectin can be used for the prevention and treatment of COVID-19. As recently discussed in BJCP, this highlights the critical need to consider pharmacological principles to guide in vitro testing when repurposing old drugs for therapeutic use against COVID-199.

\section{CONFLICT OF INTEREST}

None

\section{REFERENCES}

1. Caly L, Druce JD, Catton MG, Jans DA, Wagstaff KM. The FDA-approved drug ivermectin inhibits the replication of SARS-CoV-2 in vitro. Antiviral Res. 2020;178:104787.

2. Wagstaff KM, Rawlinson SM, Hearps AC, Jans DA. An AlphaScreen(R)-based assay for high-throughput screening for specific inhibitors of nuclear import. J Biomol Screen.2011;16(2):192-200.

3. Smit MR, Ochomo EO, Waterhouse D, et al. Pharmacokinetics-Pharmacodynamics of High-Dose Ivermectin with Dihydroartemisinin-Piperaquine on Mosquitocidal Activity and QT-Prolongation (IVERMAL). Clin Pharmacol Ther.2019;105(2):388-401.

4. Duthaler U, Suenderhauf C, Karlsson MO, et al. Population pharmacokinetics of oral ivermectin in venous plasma and dried blood spots in healthy volunteers. Br J Clin Pharmacol.2019;85(3):626-633.

5. Guzzo CA, Furtek CI, Porras AG, et al. Safety, tolerability, and pharmacokinetics of escalating high doses of ivermectin in healthy adult subjects. J Clin Pharmacol. 2002;42(10):1122-1133.

6. Baraka OZ, Mahmoud BM, Marschke CK, Geary TG, Homeida MM, Williams JF. Ivermectin distribution in the plasma and tissues of patients infected with Onchocerca volvulus. Eur J Clin Pharmacol.1996;50(5):407-410.

7. Weil GJ, Bogus J, Christian M, et al. The safety of double- and triple-drug community mass drug administration for lymphatic filariasis: A multicenter, open-label, cluster-randomized study. PLoS Med.2019;16(6):e1002839.

8. Solomon S. FDA Letter to Stakeholders: Do Not Use Ivermectin Intended for Animals as Treatment for COVID-19 in Humans. 2020; https://www.fda.gov/animal-veterinary/product-safety-information/fda-letterstakeholders-do-not-use-ivermectin-intended-animals-treatment-covid-19-humans. Accessed April 15th, 2020.

9. Smith PF, Dodds M, Bentley D, Yeo K, Rayner C. Dosing will be a key success factor in repurposing antivirals for COVID-19. Br J Clin Pharmacol. 2020.

\section{FIGURE LEGEND}

Figure 1: Maximal concentrations of ivermectin in plasma of treated subjects. Data pooled from refs ${ }^{3-5}$. When necessary, an estimated body weight of $70 \mathrm{~kg}$ was used for calculations. Note that none of the doses reached the $5 \mu \mathrm{M}$ concentration required for the antiviral effect of ivermectin (dotted line). 
Ivermectin $\mathrm{C}_{\max }$ in Clinical Studies

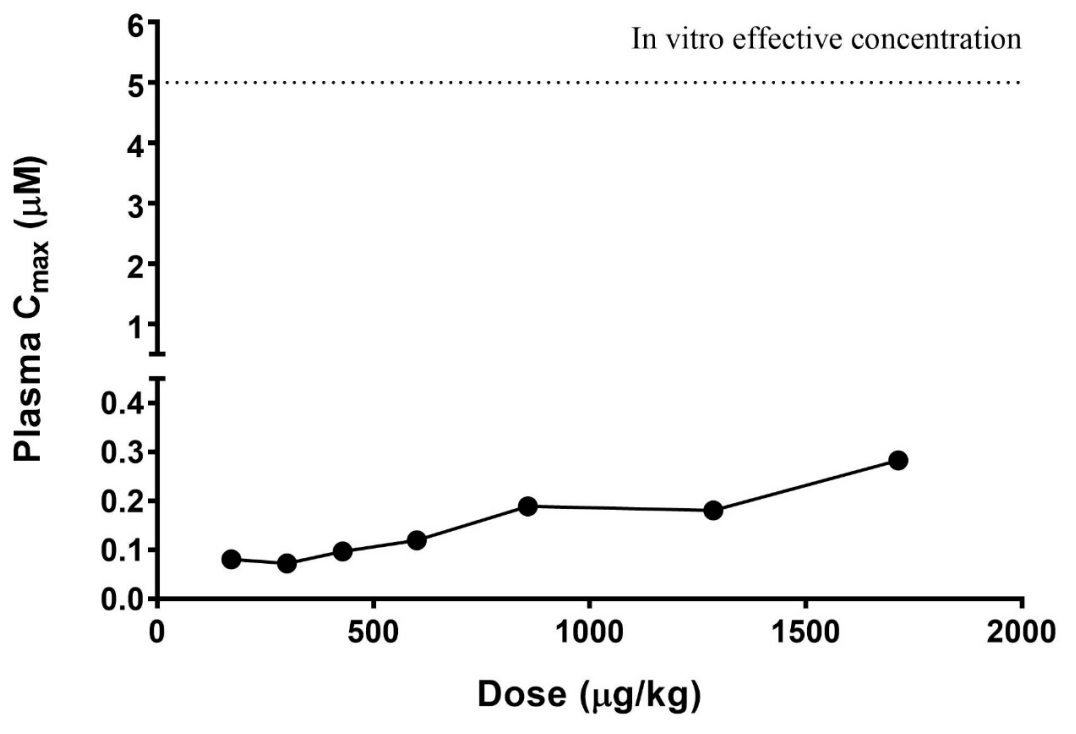

